\title{
GENERATING SYNTHETIC IRISES BY FEATURE AGGLOMERATION
}

\author{
Samir Shah and Arun Ross \\ West Virginia University, Morgantown, WV, USA. \\ sshah@csee.wvu.edu, arun.ross@mail.wvu.edu
}

\begin{abstract}
We propose a technique to create digital renditions of iris images that can be used to evaluate the performance of iris recognition algorithms. The proposed scheme is implemented in two stages. In the first stage, a Markov Random Field model is used to generate a background texture representing the global iris appearance. In the next stage a variety of iris features, viz., radial and concentric furrows, collarette and crypts, are generated and embedded in the texture field. The iris images synthesized in this manner are observed to bear close resemblance to real irises. Experiments confirm the potential of this scheme to generate a database of synthetic irises that can be used to evaluate iris recognition algorithms.
\end{abstract}

Index Terms - Biometrics, Synthetic iris, Feature agglomeration, Markov Random Fields (MRF)

\section{INTRODUCTION}

The iris is an internal organ of the eye that is located between the cornea and the lens. The primary function of the iris is to regulate the size of the pupil, which in turn controls the amount of light entering the eye. This is made possible by the presence of a sphincter muscle and a set of dilator muscles, that are connected by a pigmented fibrovascular tissue known as the stroma. These muscles appear as radial and circular furrows on the stroma. From an anatomical perspective, the iris is a multilayered structure consisting of "pectinate ligaments adhering into a tangled mesh revealing striations, ciliary processes, crypts, rings, furrows, a corona, sometimes freckles, vasculature, and other features [1]. The richness and stability of the textural characteristic of the iris makes it a very powerful biometric which can be used to distinguish even identical twins. A near-infrared lighting source is typically used to highlight the textural intricacies of the iris.

The iris is divided into two regions: the pupillary zone or the inner region which is located closer to the pupil, and the ciliary zone or the outer region which comprises the rest of the iris (Figure 1). These two regions are separated by the collarette which typically represents the thickest part of the human iris. The iris also contains sharply demarcated crypts that are a result of iris thinning which exposes the darkly pigmented posterior layer. Thus, the iris in the presence of near- infrared lighting, is observed to have several features including radial and concentric furrows, crypts, collarette and the pupil, all of which contribute to its uniqueness and play a significant role in identifying an individual.

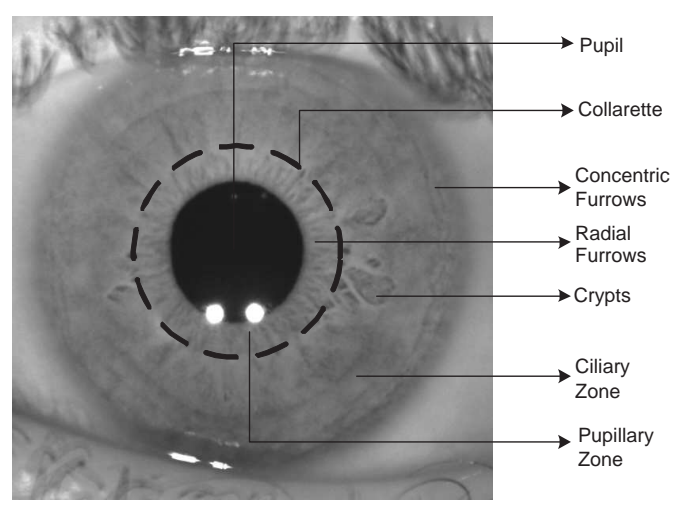

Fig. 1. Anatomy of the iris (the white dots near the pupil are the specular reflections).

The biometric literature is now replete with techniques that extract information from iris images in order to represent and match irises ( [1], [2], [3]). Although most existing iris recognition algorithms claim a very low False Accept Rate, only Daugman's algorithm [1] has been tested on a large proprietary database containing about 799, 566 iris images [4]. There are very few publicly available iris databases. Further, the ones that are available contain data pertaining to a limited number of individuals (Table 1). With the emergence of new iris recognition algorithms, it is imperative that a large database is publicly available in order to evaluate and compare these algorithms on a common platform. Building a large iris database is difficult because the process of data acquisition itself is time consuming and expensive. Moreover, privacy concerns associated with collecting and publicly disseminating biometric images have prevented several organizations from sharing images acquired in real-world operational scenarios. Due to these constraints, the possibility of generating synthetic iris databases is an attractive alternative. Some iris synthesis algorithms have been proposed in the literature. Cui et al. [5] propose an iris synthesis method using Principal Component Analysis (PCA) and super-resolution. Makhtal and Ross [6] use Markov Random Field (MRF) mod- 


\begin{tabular}{|c|c|c|c|}
\hline Database & Eyes & Images/eye & Total images \\
\hline CASIA v1.0 [9] & 108 & 7 & 756 \\
\hline UBIRIS [10] & 241 & Variable & 1877 \\
\hline UPOL [11] & 128 & 3 & 384 \\
\hline MMU [12] & 90 & 5 & 450 \\
\hline
\end{tabular}

Table 1. Examples of publicly available iris databases.

eling to generate iris-like texture from a set of primitive elements. Zuo and Schmid [7] employ a three dimensional coordinate system to generate the stroma which is then suitably modified to include the other elements of the iris. Lefohn et al. [8] create an artificial iris by stacking several semitransparent layers with each layer embedding one or more components of the human iris.

In this work, we discuss a new technique for generating synthetic iris images. Here, the background texture is first generated using a texture synthesis scheme [6] based on a single primitive element (a $30 \times 30$ patch of an arbitrarily chosen iris image from the CASIA [9] database). Then, features of the iris such as the radial and concentric furrows, collarette and crypts are added to the synthetic images. Line integral convolution is used to impart texture to the radial furrows. Hence, feature agglomeration and line integral convolution are the salient components of the proposed scheme which distinguishes it from previously proposed iris synthesis schemes. The synthetic irises are validated by comparing their genuine and impostor distributions against those originating from real images. Also, a clustering procedure is used to demonstrate the similarity between real and synthetic iris images.

\section{PROCEDURE FOR IRIS SYNTHESIS}

The proposed iris synthesis algorithm can be divided into two stages: (1) Background texture synthesis using Markov Random Field and (2) Feature synthesis.

\subsection{Texture synthesis using Markov Random Field}

The background texture of the iris is first generated using a Markov Random Field (MRF) model ( [6], [13]). The MRF model of an image can be described as the probability distribution governing the intensity value of pixels in a specific neighborhood (i.e., clique) that is independent of all other pixels in the image. Texture primitives from a real iris $(30 \times 30$ pixels) are used to guide the synthesis process. The algorithm synthesizes a new texture image that is locally similar to the input primitive image but globally exhibits a different structure.

\subsection{Feature Synthesis}

In this stage, the various features of the iris such as radial and concentric furrows, crypts and collarette are synthesized and embedded in the texture generated in the previous stage. The number of these features and their positions are parameterized based on their occurrence in real iris images. (i) Radial furrows: To generate radial furrows in the synthetic iris, straight lines are radially constructed from the center of the pupil. Each line is then sampled at discrete points and the coordinates of these points are randomly perturbed. Periodic cubic spline curves are used to interpolate these perturbed coordinates resulting in a mesh with interlaced fibres (Figure 2 (e) and (f)). To impart texture to these radial furrows, Line Integral Convolution (LIC) is used. Consider a pixel at location $x_{0}=S\left(p_{0}\right)$ on the radial furrow. The intensity of that pixel is computed using one dimensional filtering as $I\left(x_{0}\right)=\int_{p_{0}-L}^{p_{0}+L} k\left(p-p_{0}\right) T S(p) d p$, where, $T$ is a Gaussian noise image and the kernel, $k$, is a one-dimensional low-pass filter of size $L=25$ pixels. The application of the LIC procedure, imparts texture to the radial furrows.

(ii) Collarette: The collarette appears as a zig-zag circumferential ridge around the pupil (Figure 1). In order to create a collarette in the synthesized iris, a circle whose radius is 20 to 30 pixels larger than the pupil is generated around the pupil. This circle is then sampled randomly at discrete points and the coordinates of these points are randomly perturbed. Periodic cubic spline curves are once again used to interpolate these coordinates resulting in a smooth random curve representing the shape of the collarette. Only furrows present within the collarette curve are retained (Figure $2(\mathrm{~g})$ ).

(iii) Concentric Furrows: Concentric furrows are circular in appearance and are concentric with the pupil. They typically appear in the ciliary area (Figure 1) and are darker in intensity than the background texture. To create concentric furrows, curves representing portions of circles whose radii are 10 to 20 pixels smaller than the iris radius are generated in the ciliary region. The region traversed by these curves are made darker in intensity and merged with the background texture (Figure 2(h)).

(iv) Crypts: The shape and number of crypts vary across irises. To generate crypts, a random number of circles ( 1 to 10) of radius 2 to 6 pixels are generated on the periphery of the collarette. Each circle is sampled at discrete points and the corresponding coordinates are randomly perturbed. A periodic cubic spline is then used to interpolate these points. The interpolated curve represents the shape of a single crypt. The pixels inside the crypt are assigned a random intensity value that is lower than the value of the surrounding background texture. To merge the generated crypts with the background, a smoothing filter is applied in the region of interest (ROI) so that its intensity blends with that of the background texture. Multiple crypts are generated at random positions in the ciliary region of the synthetic iris (Figure 2(i)). Figure 2 summarizes the various steps of the synthesis algorithm.

\section{EXPERIMENTAL RESULTS}

The proposed synthesis scheme was used to generate a total of 108 different classes of irises. The inter-class variability between these irises is due to the random feature synthesis and agglomeration process. Although these features are gen- 
星

(a)

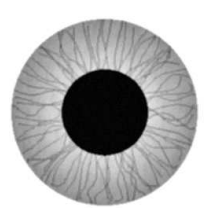

(f)

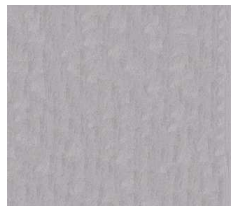

(b)

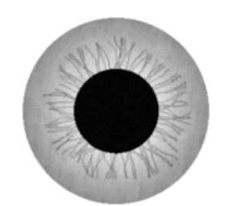

(g)

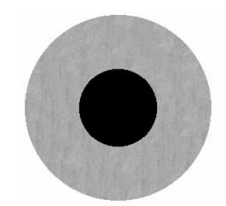

(c)

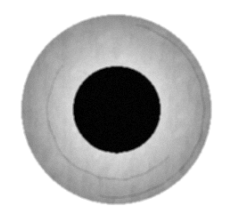

(h)

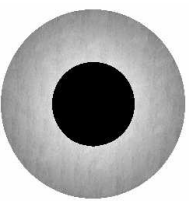

(d)

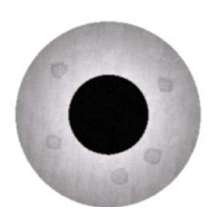

(i)

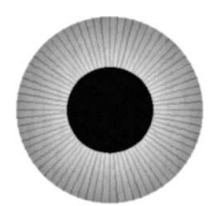

(e)

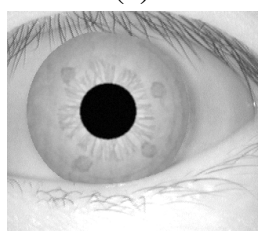

(j)

Fig. 2. Iris synthesis process. (a) Primitive; (b) Synthesized texture using MRF; (c) Carved out circular iris; (d) Iris with radial gradient; (e) Linear furrows; (f) Deformed furrows using periodic cubic splines; (g) Radial furrows circumscribed by collarette; (h) Iris with concentric furrows; (i) Iris with crypts; (j) The final synthesized iris with sclera, eyelids and eyelashes.

erated in a random fashion, their appearance and occurrences are parameterized based on similar features in real iris images. In order to introduce intra-class variability (to obtain multiple samples of each iris), the generated synthetic irises were subjected to the following operations: (i) a random rotation in the $[-15,15]$ interval (degrees); (ii) a blurring operation using pixel averaging; (iii) addition of Gaussian noise over the entire image; and (iv) changing the pupil size by an additive factor sampled from the $[-10,10]$ interval (pixels). This resulted in 7 images per class. Figure 5 shows a few synthetic irises generated by this procedure. A simple clustering experiment was performed to show that the generated synthetic iris images are similar to real iris images and different from other types of irregular stochastic texture patterns taken from the Brodatz texture library. Co-occurrence matrix, which is used vastly in the field of texture classification and analysis, captures the second order statistics of the image. In our experiment, the co-occurrence matrix was computed for each image at various displacement vector values (see [6]). A total of 111 co-occurrence matrices were considered. Four properties of the co-occurrence matrix, viz., entropy, energy, contrast and homogeneity were computed. These four properties were extracted from all the co-occurrence matrices resulting in a 444-dimensional feature vector. These feature vectors were extracted from 100 iris images in the CASIA dataset, 100 iris images in the synthetic dataset and 100 texture images in the Brodatz library. Unsupervised K-means clustering was performed using the extracted feature vectors $(\mathrm{K}=2)$. In this experiment, $99 \%$ of the real irises, $100 \%$ of the synthetic irises and $30 \%$ of the Brodatz images were clustered in a single class while $70 \%$ of the Brodatz textures along with $1 \%$ of the real irises were clustered in the other class. This suggests the textural similarity between the real and synthetic iris images.

In order to further experiment with the synthetic images, a suitable representation and matching scheme was essential.
Masek's technique [14], which is a variation of Daugman's algorithm, was used to quantify and represent the textural content of the iris images. This process entails the application of a bank of Gabor filters on an iris image and examining the phase of the resulting response at regular intervals in the image. The phase response is viewed as a binary information and, thus, each iris is represented using a string of 0's and 1's known as the iriscode. The dissimilarity between two iris images is measured by employing the hamming distance metric that outputs a match score upon comparing two such binary strings. The experiments described below utilize the synthetic irises generated by the proposed method and the real irises obtained from the CASIA database ${ }^{1}$.

In the first experiment, three different impostor score distributions were plotted. These correspond to the impostor scores based on (a) comparing synthetic images only, (b) comparing real images only, and (c) comparing real images against synthetic images. From the distributions in Figure 3, it is clear that the impostor distributions are nearly the same for all the three cases. This suggests that the proposed method is able to capture the inter-class variability commonly observed in real images. In the second experiment, the genuine and impostor distributions corresponding to the real and synthetic irises were plotted (Figure 4). This graph also suggests that the intra-class and inter-class dynamics observed in real images are captured by the feature generation process.

\section{SUMMARY AND FUTURE WORK}

In this paper, a novel technique for generating synthetic iris images has been presented. The technique first generates background texture using Markov Random Fields and then embeds individual features of the iris such as radial and concentric furrows, crypts and the collarette on the underlying texture. The synthetic irises generated using this technique

\footnotetext{
${ }^{1}$ These experiments may be repeated with other publicly available iris databases also.
} 


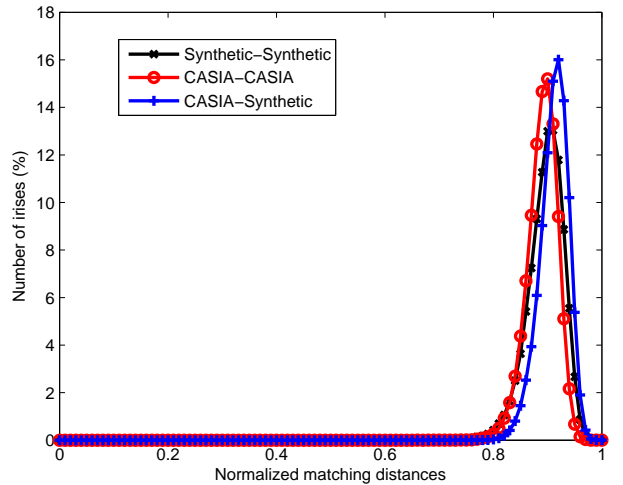

Fig. 3. Impostor distributions for real and synthetic images.

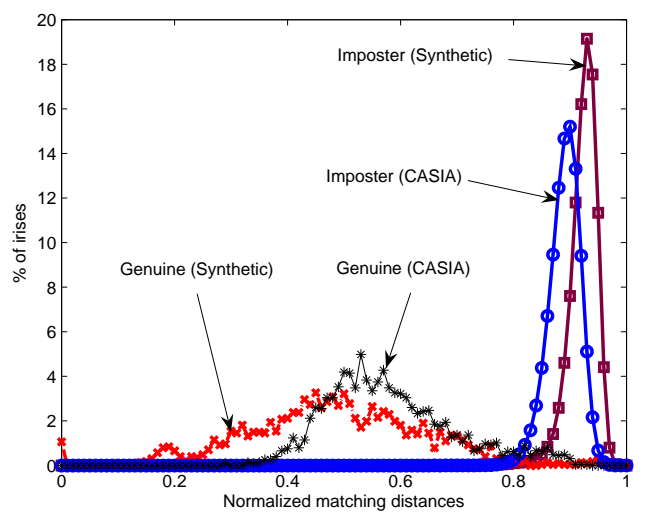

Fig. 4. Genuine and impostor match score distributions of real and synthetic images.

are observed to be similar to real iris images as demonstrated by the clustering experiment in which the synthetic irises are categorized alongside real iris images. Currently, the texture synthesis algorithm terminates after a predetermined number of iterations and, hence, we are developing techniques to dynamically determine if the desired texture has been generated. We are also working on developing measures of realism that could mathematically establish the similarity between real and synthetic iris images. Also, we are attempting to generate synthetic irises whose iriscode is similar to a (selected) real iris image.

\section{ACKNOWLEDGEMENTS}

Thanks to Dr. Yunhong Wang for granting us access to the CASIA iris database. This work was funded by the Center for Identification Technology Research (CITeR) at West Virginia University.

\section{REFERENCES}

[1] J. Daugman, "Demodulation by complex-valued wavelets for stochastic pattern recognition," International Journal of Wavelets, Multi-resolution and Information Processing, vol. 1, no. 1, pp. 1-17, 2003.
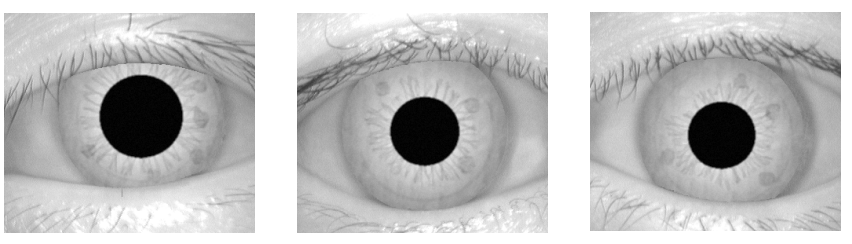

Fig. 5. Examples of synthetic iris images.

[2] R. P. Wildes, "Iris recognition: An emerging biometric technology," Proc. of the IEEE, vol. 85, no. 9, pp. 13481363, Sep. 1997.

[3] L. Ma, T. Tan, and Y. Wang, "Efficient iris recognition by characterizing key local variations," IEEE Trans. on Image Processing, vol. 13, no. 6, pp. 739-750, Jun. 2004.

[4] M. Almualla, "The UAE iris expellees tracking and border control system," Biometric Consortium Conference, September 2005, Virginia, VA.

[5] J. Cui, Y. Wang, J. Huang, T. Tan, and Z. Sun, "An iris image synthesis method based on PCA and superresolution," Proc. of ICPR, vol. 4, pp. 471-474, Aug. 2004.

[6] S. Makhtal and A. Ross, "Synthesis of iris images using Markov Random Fields," Proc. of 13th EUSIPCO, Sep. 2005.

[7] J. Zuo and N. Schmid, "A model based, anatomy based method for synthesizing iris images," in Proc. of the ICB, Hong Kong, January 2006, pp. 428-435.

[8] A. Lefohn, B. Budge, P. Shirley, R. Caruso, and E. Reinhard, "An ocularist's approach to human iris synthesis," IEEE Computer Graphics and Applications, vol. 23, no. 6, pp. 70-75, Nov./Dec. 2003.

[9] "The CASIA iris image database," Available at: http://www.sinobiometrics.com.

[10] H. Proena and L. A. Alexandre, "UBIRIS: A noisy iris image database," in Proceed. of ICIAP 2005 - Intern. Confer. on Image Analysis and Processing, 2005, vol. 1, pp. 970-977.

[11] M. Dobes and L. Machala, "UPOL iris image database," Available at: http://phoenix.inf.upol.cz/iris/.

[12] "The MMU1 iris image database," Available at: http://pesona.mmu.edu.my/ ccteo/.

[13] L. Y. Wei, Texture Synthesis by Fixed Neighborhood Searching, Ph.D. thesis, Stanford University, 2001.

[14] L. Masek and P. Kovesi, "Biometric identification system based on iris patterns," Matlab source code, The School of Computer Science and Software Engineering, The University of Western Australia, 2003. 